\title{
Acute Renal Failure due to Thrombotic Microangiopathy in Patient with Scleroderma: Autopsy Case Report
}

\author{
Akihiro Ishizu, MD, PhD, ${ }^{1}$ Shinji Fukaya, ${ }^{2}$ Utano Tomaru, ${ }^{2}$ Kazuaki Katsumata, ${ }^{1}$ \\ Akira Suzuki, ${ }^{3}$ Yuka Umemoto, ${ }^{4}$ Akira Furusaki,${ }^{4}$ and Yoshiharu Amasaki ${ }^{4}$
}

\begin{abstract}
This study presents a patient who died of acute renal failure (ARF) as a complication of scleroderma. The patient remained normotensive throughout the clinical course. Myeloperoxidase-anti-neutrophil cytoplasmic antibody was negative. Autopsy revealed fibrin thrombi in the glomerular capillaries and afferent arterioles, mesangiolysis, and double contour of the glomerular basement membrane. Contrarily, "onionskin lesions" of renal interlobular arteries, the histological hallmark of scleroderma renal crisis, were not discovered. These findings suggested that thrombotic microangiopathy (TMA) was the cause of ARF. Although the frequency is not high, close monitoring should be given to TMA in scleroderma because of possible mortality.
\end{abstract}

Keywords: cleroderma, acute renal failure, thrombotic microangiopathy

\section{INTRODUCTION}

Ccleroderma (systemic sclerosis) is an autoimmune disease with unknown etiology characterized by progressive fibrosis of the skin and visceral organs. ${ }^{1)}$ Females are affected more predominantly than males. The incidence of the disease peaks in the third to fifth decade of life. Renal involvement is a major complication of scleroderma. ${ }^{2)}$ Scleroderma renal crisis (SRC) is almost invariably fatal, with most patients dying within 3 months of presentation from the so-called "malignant hypertension." The histological hallmark of SRC is rec-

${ }^{1}$ Faculty of Health Sciences, Hokkaido University, Sapporo, Hokkaido, Japan

${ }^{2}$ Department of Pathology, Hokkaido University Graduate School of Medicine, Sapporo, Hokkaido, Japan

${ }^{3}$ Department of Pathology, Konan Hospital, KKR Sapporo Medical Center, Sapporo, Hokkaido, Japan

${ }^{4}$ The Center for Rheumatic Diseases, Tonan Hospital, KKR Sapporo Medical Center, Sapporo, Hokkaido, Japan

Received: September 3, 2012; Accepted: September 22, 2012

Corresponding author: Akihiro Ishizu, MD, PhD. Faculty of Health Sciences, Hokkaido University, Kita-12, Nishi-5, Kita-ku, Sapporo, Hokkaido 060-0812, Japan

Tel: +81-11-706-3385, Fax: +81-11-706-4916

E-mail: aishizu@med.hokudai.ac.jp ognized as "onionskin lesions" of the renal interlobular arteries. ${ }^{3)}$ Although SRC is the most prominent renal manifestation of scleroderma, there are other important complications, including crescentic glomerulonephritis associated with myeloperoxidase-anti-neutrophil cytoplasmic autoantibody (MPO-ANCA) and thrombotic microangiopathy (TMA). ${ }^{4,5)}$

\section{Case Report}

A 45-year-old woman was admitted to the hospital due to fever, general fatigue, and respiratory distress, which started to manifest 2 weeks prior to admission. She was diagnosed with rheumatoid arthritis 3 years ago, but the activity of arthritis was controlled to clinical remission by oral administration of methotrexate $(6 \mathrm{mg} /$ week). Upon admission (day 1), the vital signs were within normal limits with a blood pressure of 126/76 $\mathrm{mmHg}$. Physical examination revealed stiffness of the skin of the distal extremities and around the mouth, and fine crackles on auscultation of the thorax. Although Raynaud's phenomenon was unrecalled when inquired, sclerodactyly with fingertip ulcer scar suggested the development of scleroderma. Blood count revealed leukocytosis $(23030 / \mu \mathrm{L})$ with predominance of neutrophils, 
anemia $\left(347 \times 10^{4} / \mu \mathrm{l}\right)$ with $9.2 \mathrm{~g} / \mathrm{dl}$ of hemoglobin, and thrombocytopenia $\left(6.1 \times 10^{4} / \mu 1\right)$. Serum albumin level was $2.4 \mathrm{~g} / \mathrm{dl}$. The direct and indirect bilirubin levels were 0.7 and $1.7 \mathrm{mg} / \mathrm{dl}$, respectively. The level of lactate dehydrogenase was remarkably elevated (1169 IU/L), though the transaminase levels were not so high (AST: $69 \mathrm{IU} / \mathrm{L}$, ALT: $40 \mathrm{IU} / \mathrm{L})$. Although the levels of blood urea nitrogen (BUN) and creatinine were $23 \mathrm{mg} / \mathrm{dL}$ and $0.68 \mathrm{mg} / \mathrm{dL}$, respectively, both microscopic hematuria and proteinuria were already present at this time. Fibrin/fibrinogen degradation products were increased in the serum $(15.9 \mathrm{mg} /$ $\mu \mathrm{L})$. Serological examination revealed a high level of C-reactive protein $(18.4 \mathrm{mg} / \mathrm{dL})$. Antinuclear antibodies (homogeneous and speckled pattern) were positive (1:80), but other autoantibodies, including anti-DNA antibodies, anti-RNA polymerase antibodies, anti-Scl-70 antibodies, anti-phospholipid antibodies, and MPO-ANCA, were negative. Chest $\mathrm{CT}$ revealed mild interstitial pneumonitis (IP) and minimal pericardial effusion. Collective findings suggested that the patient developed limited cutaneous type scleroderma with IP and pericarditis. The complication of thrombotic thrombocytopenic purpura (TTP) was also considered because of thrombocytopenia and probable hemolytic anemia. Based on the diagnosis, administration of corticosteroids (30 mg/day) was started, and 5 units of fresh-frozen plasma were transfused.

Chest discomfort was experienced the next morning (day 2). The platelet count further decreased to $3.6 \times 10^{4} / \mu \mathrm{L}$, and the levels of BUN and creatinine were elevated to $34 \mathrm{mg} / \mathrm{dL}$ and $0.94 \mathrm{mg} / \mathrm{dL}$, respectively. Hyperkalemia $(6.3 \mathrm{mEq} / \mathrm{L})$ also appeared at this time. Fragmentation of red blood cells (RBCs) was demonstrated on peripheral blood smear; thereby, the presence of hemolysis was implied. Although ADAMTS13 activity remained unknown at this point, the complication of TTP was considered. Therefore, plasmapheresis and hemodialysis were conducted. However, from this point in time, the patient became unconscious.

The levels of BUN and creatinine were further elevated to $57 \mathrm{mg} / \mathrm{dl}$ and $1.92 \mathrm{mg} / \mathrm{dl}$, respectively, and urinary volume gradually diminished over the next day (day 3). She subsequently died of acute renal failure (ARF) followed by multiple organ failure 2 days after admission. Throughout the clinical course, her blood pressure did not rise to hypertensive level. After her death, ADAMTS13 activity was demonstrated to be within normal limits.

Autopsy revealed that the weights of the kidneys were within the normal range (right, $170 \mathrm{~g}$; left, 195g). However, fibrin thrombi were frequently seen in the glomerular cap- illaries (Fig. 1a and 1b) and afferent arterioles (Fig. 1c). Moreover, mesangiolysis (Fig. 1d) and double contour of the GBM (Fig. 1e) were observed. On the other hand, overt onionskin lesions of the renal interlobular arteries were not discovered (Fig. 1f). These findings suggested that the patient developed TMA.

Autopsy findings in other organs were as follows: mild, nonspecific IP in the lungs; pericarditis with serous pericardial effusion $(100 \mathrm{~mL})$; erosion of the lower part of the esophagus; patchy liver necrosis; and slight congestion of the spleen. Nonspecific IP, pericarditis (serositis), and esophageal erosion might be associated with scleroderma, and the liver injury and splenic congestion might be related to TMA. There was no infectious focus in any organ.

\section{Discussion And Conclusion}

In the patient, the clinical and histological findings of hemolysis with fragmentation of RBCs, fibrin thrombi in the glomerular capillaries and afferent arterioles, mesangiolysis, and double contour of the GBM are consistent with TMA. The concept of TMA includes a broad spectrum of diseases, such as TTP, hemolytic uremic syndrome (HUS), and disseminated intravascular coagulation (DIC). ${ }^{6}$ In typical TTP, the activity of ADAMTS13 is low due to mutation of the gene (congenital TTP) or generation of inhibitory antibody (acquired TTP); whereas, it remains within normal limits in HUS and DIC.7) In the patient, ADAMTS13 activity remained within normal level; thus, typical TTP could be ruled out. On the other hand, it is known that the major cause of HUS is an infection by Shiga-toxin producing Escherichia coli $\left.\mathrm{O} 157 .{ }^{6}\right)$ Since there was no evidence of such infection in the patient, Shiga-toxin-mediated HUS could also be ruled out. However, the elaboration of the differential diagnoses (TTP, HUS, and DIC) is occasionally difficult. Thus, the patient was diagnosed with TMA in a broad sense. Other etiologies of TMA include connective tissue diseases, malignancy, pregnancy, transplantation, and drugs. ${ }^{6)}$ Among them, scleroderma is considered as the cause of TMA in the patient. Although the brain was not examined in autopsy, the involvement of the central nervous system in TMA could be the cause of the rapid deterioration of the patient's level of consciousness on day 2.

The principal pathogenesis of TMA has been regarded as injury of small vessel endothelial cells. Recent study has revealed that excessive production of endothelin-1 is critically associated with such endothelial cell injury. ${ }^{8}$ 

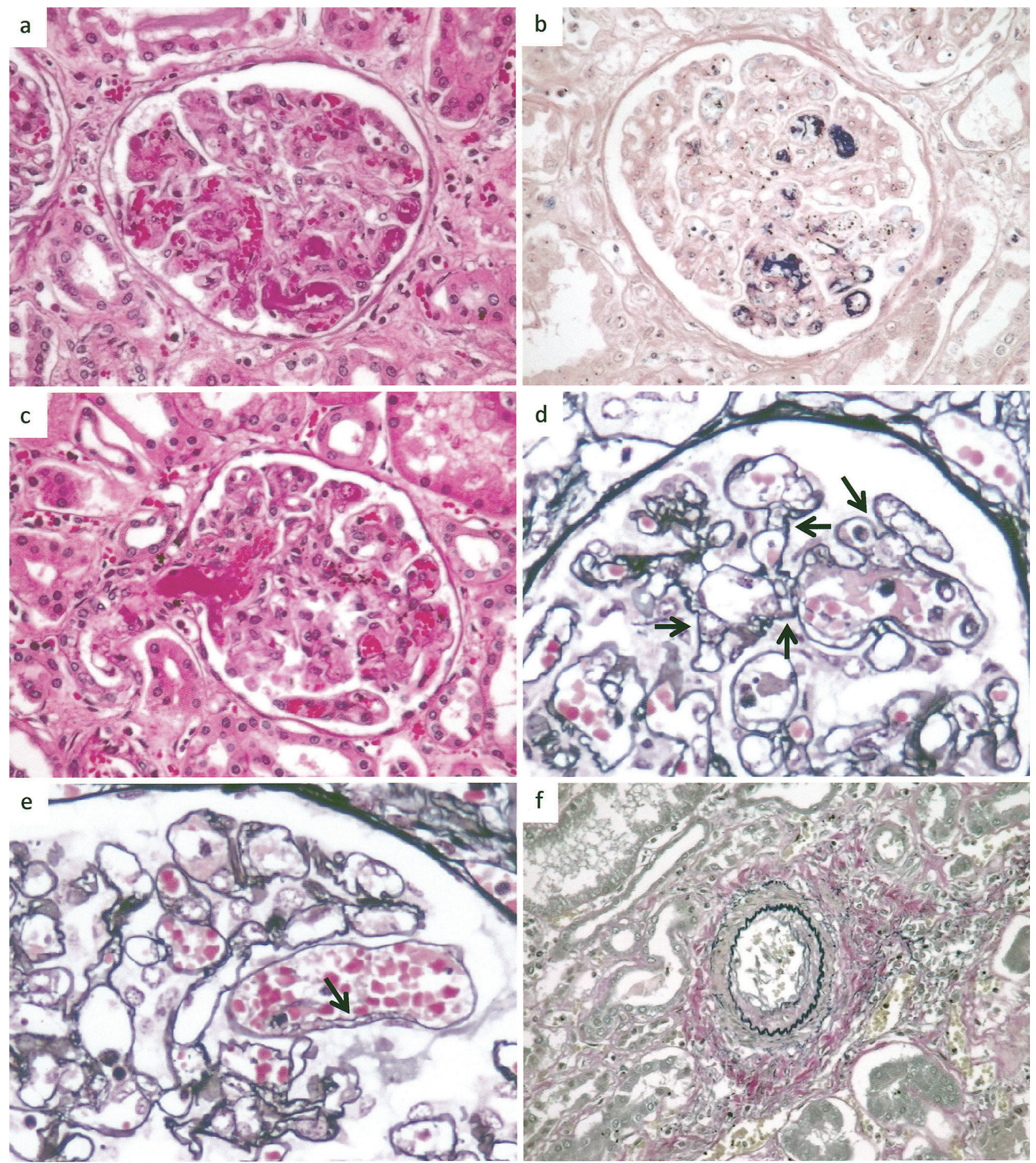

Fig. 1 Fibrin thrombi in the glomerular capillaries (a: HE, b: PTAH staining) and afferent arteriole (c: HE). Original magnification: $\times 200$. Mesangiolysis (d: PAM/HE staining, arrows). Original magnification: $\times 400$. Double contour of the GBM (e: PAM/HE staining, arrow). Original magnification: $\times 400$. Onion-skin lesions are not seen in the renal interlobular arteries (f: EVG staining). Original magnification: $\times 200$.

The process leads to accumulation of platelets in the endothelial injury sites followed by the formation of fibrin thrombi. As a result, platelets are consumed, and RBCs are mechanically destroyed. Detachment of glomerular endothelial cells results in mesangiolysis and subsequent duplication of the GBM. ${ }^{9)}$ Circulating endothelin-1 lev- els are reported to be high in scleroderma patients with vascular manifestations. ${ }^{10)}$ Therefore, an increase in the serum endothelin-1 level is considered a possible cause of TMA in scleroderma. It would have been ideal if the serum level of endothelin-1 were known; however, patient data were not available. 
Regardless of etiology, ARF is a medical emergency that requires careful management. Although TMA is a relatively rare complication in scleroderma, close monitoring should be conducted because of risk of patient demise.

\section{Disclosure Statement}

The authors have declared that no conflict of interest exists.

1) Chifflot H, Fautrel B, Sordet $C$, et al. Incidence and prevalence of systemic sclerosis: a systematic literature review. Semin Arthritis Rheum 2008; 37: 223-35. [Medline] [CrossRef]

2) Penn H, Denton CP. Diagnosis, management and prevention of scleroderma renal disease. Curr Opin Rheumatol 2008; 20: 692-6. [Medline] [CrossRef]

3) Penn H, Howie AJ, Kingdon EJ, et al. Scleroderma renal crisis: patient characteristics and long-term outcomes. QJM 2007; 100: 485-94. [Medline] [CrossRef]

4) Arad U, Balbir-Gurman A, Doenyas-Barak K, et al. Anti-neutrophil antibody associated vasculitis in systemic sclerosis. Semin Arthritis Rheum 2011; 41:
223-9. [Medline] [CrossRef]

5) Abudiab M, Krause ML, Fidler ME, et al. Differentiating scleroderma renal crisis from other causes of thrombotic microangiopathy in a postpartum patient. Clin Nephrol DOI: 10.5414/CN107465. [CrossRef] [Medline]

6) Radhi M, Carpenter SL. Thrombotic microangiopathies. ISRN Hematol 2012; 2012: 310596. [CrossRef] [Medline]

7) Furlan M, Robles R, Galbusera M, et al. von Willebrand factor-cleaving protease in thrombotic thrombocytopenic purpura and the hemolytic-uremic syndrome. N Engl J Med 1998; 339: 1578-84. [Medline] [CrossRef]

8) Mouthon L, Mehrenberger M, Teixeira L, et al. Endothelin-1 expression in scleroderma renal crisis. Hum Pathol 2011; 42: 95-102. [Medline] [CrossRef]

9) Changsirikulchai S, Myerson D, Guthrie KA, et al. Renal thrombotic microangiopathy after hematopoietic cell transplant: role of GVHD in pathogenesis. Clin J Am Soc Nephrol 2009; 4: 345-53. [Medline] [CrossRef]

10) Vancheeswaran R, Magoulas T, Efrat G, et al. Circulating endothelin-1 levels in systemic sclerosis subsets-a marker of fibrosis or vascular dysfunction? J Rheumatol 1994; 21: 1838-44. [Medline] 